\title{
A permanent neurosurgical challenge: low grade gliomas
}

\author{
Daniel Mihai Teleanu ${ }^{1}$, Nicolae-Stefan Bogaciu ${ }^{2}$, Andreea Marinescu ${ }^{3}$, \\ Raluca Ioana Teleanu ${ }^{4}$, Adrian Balasa ${ }^{5}$ \\ ${ }^{1}$ Department of Neurosurgery, Emergency University Hospital Bucharest, \\ "Carol Davila" University of Medicine and Pharmacy, Bucharest, Romania \\ ${ }^{2}$ Department of Neurosurgery, Emergency University Hospital, Bucharest, Romania \\ ${ }^{3}$ Department of Radiology, Emergency University Hospital, Bucharest, Romania \\ 4"Carol Davila" University of Medicine and Pharmacy, Bucharest, Romania \\ ${ }^{5}$ Department of Neurosurgery, Emergency Hospital, University of Medicine and Pharmacy, \\ Targu Mures, Romania
}

\begin{abstract}
Introduction. The utilization of Magnetic Resonance Spectroscopy (MRS) brings an important piece of information to an overall MR study, thus aiding the physician in making an accurate assumption regarding the histological grade of a tumour. The purpose of this study is to verify the reliability of MRS in correctly diagnosing both the nature of tumours and their grade.

Material and methods. This is an observational study that was conducted from January 2011 to June 2016 on 49 patients confirmed to be low-grade gliomas (LGG) by pathological examination, who were admitted in our Neurosurgery Department in this period. Both retrospective and prospective data were collected. Inclusion criteria comprise unique tumoral lesion at the moment of diagnosis, follow-up for at least one year. Exclusion criteria included: other types of tumors with any location, patient refusal to undergo histopathological examination of the resected tissue, uncompliant and non-collaborating patients. Of all patients, 22 were subjected to an MRS study which suggested the presence of a low-grade glioma subsequently confirmed by the pathological examination.

Results. MRS has been shown to provide accurate non-invasive diagnosis of LGG's, by analysing the concentration of metabolites inside the lesions which tend to be specifi $c$ for these tumours: relatively elevated levels of $\mathrm{N}$ acetylaspartate and creatine with reduced levels in the concentration of choline and absent lipids and lactate. It has also been observed that pre-operative MRS assists the physician in selecting the optimal place for biopsy, so that the pathological examination provides conclusive results.

Conclusions. With the increased availability of MRI technology, MRS comes as a milestone in the advancement of more accurate and patient-friendly methods of diagnostic for pathologies as LGG's which constitute a permanent challenge for neurosurgeons. The result of this study underlines the importance of MRS as a method for follow up patients with LGG, but cannot replace the need for obtaining biotic tissue for pathological examination, especially after the new grading system of WHO, which was published in June 2016. This new grading system takes into account the molecular biology in predicting the natural history of cerebral tumours.
\end{abstract}

Keywords: magnetic resonance spectroscopy, low grade glioma, management, biopsy

\footnotetext{
Abbreviations

AA - Anaplastic astrocytoma

Cho - Choline

CNS - Central Nervous System

$\mathrm{Cr}-$ Creatine

CT - Computed Tomography

GBM - Glioblastoma multiforme

Gy - Gray

HGG - High Grade Glioma

HP - Histopathological

Lac - Lactates

LGG - Low Grade Glioma
}

\author{
Lip - Lipids \\ mIns - Myo-Inositol \\ MR - Magnetic Resonance \\ MRI - Magnetic Resonance Imaging \\ MRS - Magnetic Resonance Spectroscopy \\ NAA - N-acetylaspartate \\ RT - Radiotherapy \\ SPECT - Single Photon Emission Computed Tomography \\ SVS - Single Voxel Spectroscopy \\ WHO - World Health Organization
}




\section{INTRODUCTION}

Gliomas are a type of neuroepithelial tumors that develop from glial cells. They are primarily located in the brain and, on rare occasions, in the spine. The WHO grading system classifies gliomas by assessing histological features of the tumours: necrosis, endothelial proliferation, atypical cellularity, mitoses and, as of 2016, by adding the use of molecular parameters (1). There are 4 grades for the classification of brain tumours, of which grades I-II are considered to have a more benign development, being known as low-grade gliomas. Grades III-IV tumours having a more malignant behavior, are known as high-grade gliomas. Despite the fact that both grade I and II gliomas are called lowgrade, the management for each type is different: grade I tumors are prone to surgical cure, while the management for grade II tumors is very controversial. The purposes of surgical treatment would be to obtain representative specimens that lead to an accurate diagnosis, to reduce tumour mass in order to alleviate neurological symptoms and to decrease the chance of relapse, knowing that large tumour volumes and growth rates are associated with malignant transformation. When choosing surgical treatment, one has to take into account the clinical and neurological state of the patient. The ones who would benefit the most from surgery are those with neurological deficits, depending on whether they are caused by large masses of the tumours or by disruption of the brain tissue, because a conservative approach will surely lead to neurologic deterioration. Seizure producing tumours are also candidates for surgical removal, the benefit for the patients being a better control through antiepileptic drugs (2).

Imaging studies usually performed in patients with LGGs consist mostly of MRI techniques (T1, contrast-enhanced T1, T2, FLAIR, perfusion MR etc), of which a great volume of information has been lately provided by MR spectroscopy. This technique analyzes the concentration of multiple metabolites (N-acetylaspartate, Creatine, Choline, Lactate, Lipids, Myoinositol, Alanine, Glutamate-Glutamine) in scanned brain tissue areas, providing metabolites sequences that are specific for each different type of lesion. NAA concentration declines with the rise of neuronal destruction, choline is a marker for cell membrane density and integrity and it rises proportionally with the grade of the tumour, creatine being linked to energy metabolism, will decrease with the increase of the grade, lactates and lipids suggest anaerobic metabolism and are mainly absent in low-grade gliomas. The signature for LGG is represented by relatively elevated levels of $\mathrm{N}$-acetylaspartate and creatine with reduced levels in the concentration of choline and absent lipids and lactate $(3,4)$, but of course, some atypical findings may exist. There are also some important ratios to be taken into account, of which we remember NAA/Cr, Cho/NAA and Cho/Cr (Table 1). The latter two are relatively elevated in low grade gliomas, whereas high-grade gliomas have even more elevated ratios (5).

This allows for a better evaluation of the site of needle-biopsy, where this is the preferred option for management of the tumours, so that the collected specimen can provide accurate information regarding the nature and grading of the mass (6).

The aim of this study is to show the raising importance of Magnetic Resonance Spectroscopy in the challenging management of low-grade glioma, emphasizing its usefulness regarding preoperative planning. Magnetic Resonance Spectroscopy is a non-invasive, harmless tool for obtaining precious information about the place, type and possible behaviour of brain tumours.

\section{MATERIAL AND METHODS}

For this study, we collected data about 49 patients confirmed to be low-grade gliomas (LGG) by pathological examination, admitted in our department from January 2011 to June 2016.

Inclusion criteria for this group where: histopathological diagnosis of low grade glioma using a specimen obtained either with surgical resection or stereotactic biopsy, follow-up for at least one year, unique tumoral lesion (LGG) at the time of diagnosis.

Exclusion criteria included: other types of tumors with any location, patient refusal to undergo

TABLE 1. Evolution of metabolites levels in different tumoral grades

\begin{tabular}{|l|c|c|c|c|c|c|c|c|}
\hline Grade & NAA & Cho & Cr & mIns & Lip & Lac & Cho/NAA & Cho/Cr \\
\hline I & 0 & $\uparrow$ & 0 & $\uparrow$ & 0 & 0 & $\uparrow$ & $\uparrow$ \\
\hline II & $\downarrow$ & $\uparrow$ & $\downarrow$ & $\uparrow \uparrow$ & 0 & 0 & $\uparrow \uparrow$ & $\uparrow$ \\
\hline III & $\downarrow \downarrow$ & $\uparrow \uparrow$ & $\downarrow \downarrow$ & 0 & $\uparrow$ & $\uparrow \uparrow$ & $\uparrow \uparrow \uparrow$ & $\uparrow \uparrow$ \\
\hline IV & $\downarrow \downarrow \downarrow$ & $\uparrow \uparrow \uparrow$ & $\downarrow \downarrow \downarrow$ & $\downarrow \downarrow \downarrow$ & $\uparrow \uparrow \uparrow$ & $\uparrow \uparrow \uparrow$ & $\uparrow \uparrow \uparrow$ & $\uparrow \uparrow \uparrow$ \\
\hline
\end{tabular}


TABLE 2. Signs and symptoms at admission for the patients with low grade gliomas

\begin{tabular}{|c|c|c|c|c|c|c|c|c|}
\hline $\begin{array}{l}\text { Signs/symptoms } \\
\text { on presentation }\end{array}$ & $\begin{array}{l}\text { Motor deficits } \\
\text { (monoparesis, } \\
\text { paraparesis, } \\
\text { tetraparesis) }\end{array}$ & Seizures & $\begin{array}{l}\text { Cranial } \\
\text { nerves } \\
\text { defic s }\end{array}$ & $\begin{array}{l}\text { Speech disorders } \\
\text { (aphasia, } \\
\text { dysarthria) }\end{array}$ & Headache & $\begin{array}{l}\text { Altered state of } \\
\text { consciousness }\end{array}$ & $\begin{array}{l}\text { Sensory } \\
\text { deficits }\end{array}$ & $\begin{array}{l}\text { Affec ve } \\
\text { disorder }\end{array}$ \\
\hline $\begin{array}{l}\text { Number of } \\
\text { patients }\end{array}$ & 23 & 18 & 17 & 16 & 9 & 5 & 3 & 1 \\
\hline
\end{tabular}

histopathological examination of the resected tissue, uncompliant and non-collaborating patients.

There were 28 male and 21 female patients, the youngest being 15 years old on the day the diagnosis was made, and the oldest 76 , with an average age of onset of 44 . The signs and symptoms on admission were dominated by seizures and motor deficits, as described in Table 2.

All of our patients were subjected to a brain CTscan, 38 have had an MRI, 22 of which have also had an MRS study performed and 1 patient was examined using SPECT. All types of imaging studies that analyze just the anatomy could provide only
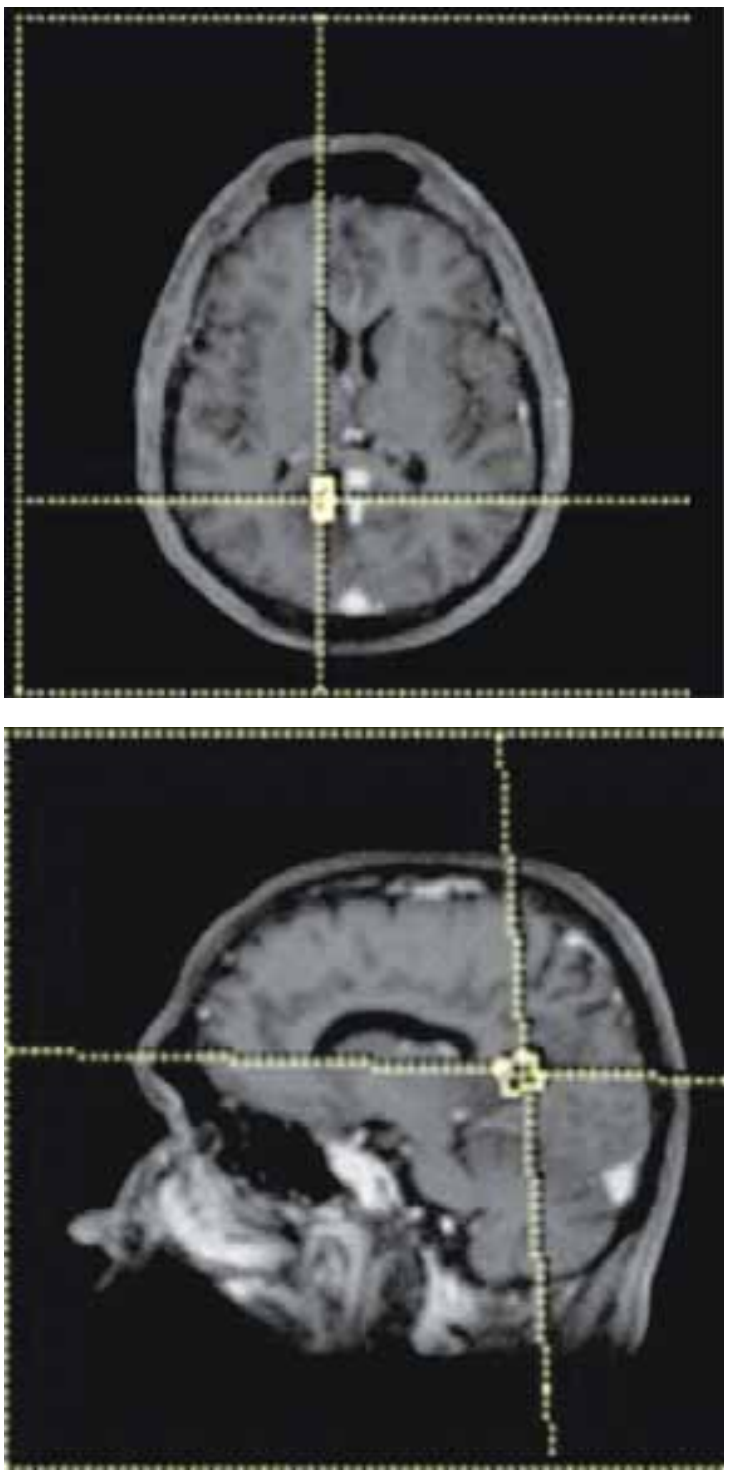

the diagnosis of an intracranial lesion or tumoral mass, without being able to distinguish its nature, whereas Spectroscopy was able to provide clues for quite an accurate diagnosis. MR Spectroscopy was performed using the single voxel spectroscopy (SVS) technique, where the technician would select a voxel from where he would acquire the signal from inside the tumoral mass. Starting from anatomical images, a voxel is constructed at the intersection of perpendicular planes in the three dimensions of space. The result is a volume of interest which can be further studied (Fig. 1). The advantages of SVS are represented by a high quality of the spectra, homogeneity of the field and swiftness of the examination (6-8).

Histopathological diagnoses were obtained either from stereotactic guided biopsy (Fig. 2), which was the case for the majority of the patients (29), or from surgical resection acquired specimens for 20 patients. For both procedures, different factors, as general and neurological status, volume and location of tumoral mass, patient's choice, were taken into consideration.
FIGURE 1. Selecting a voxel by combining planes in three dimensional space

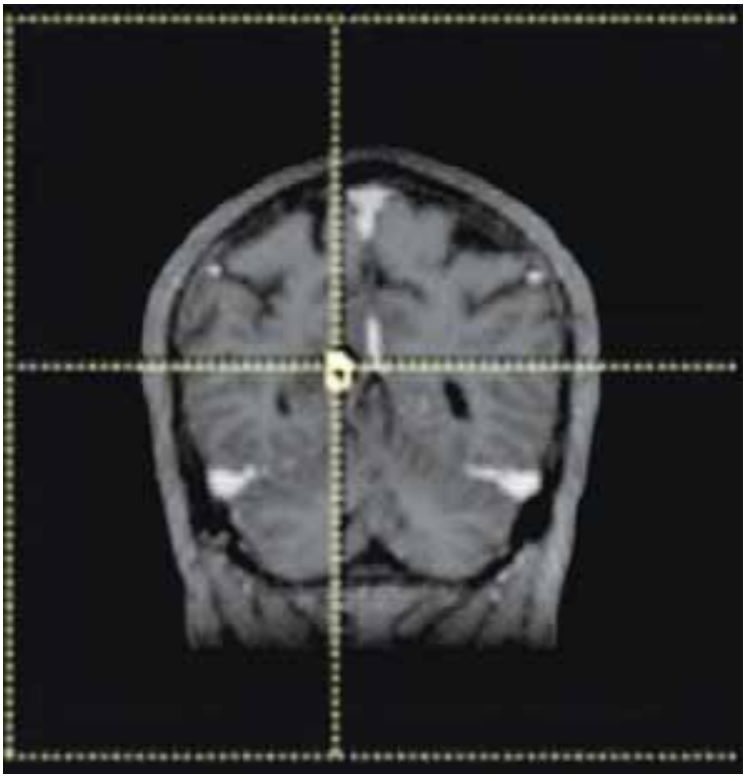



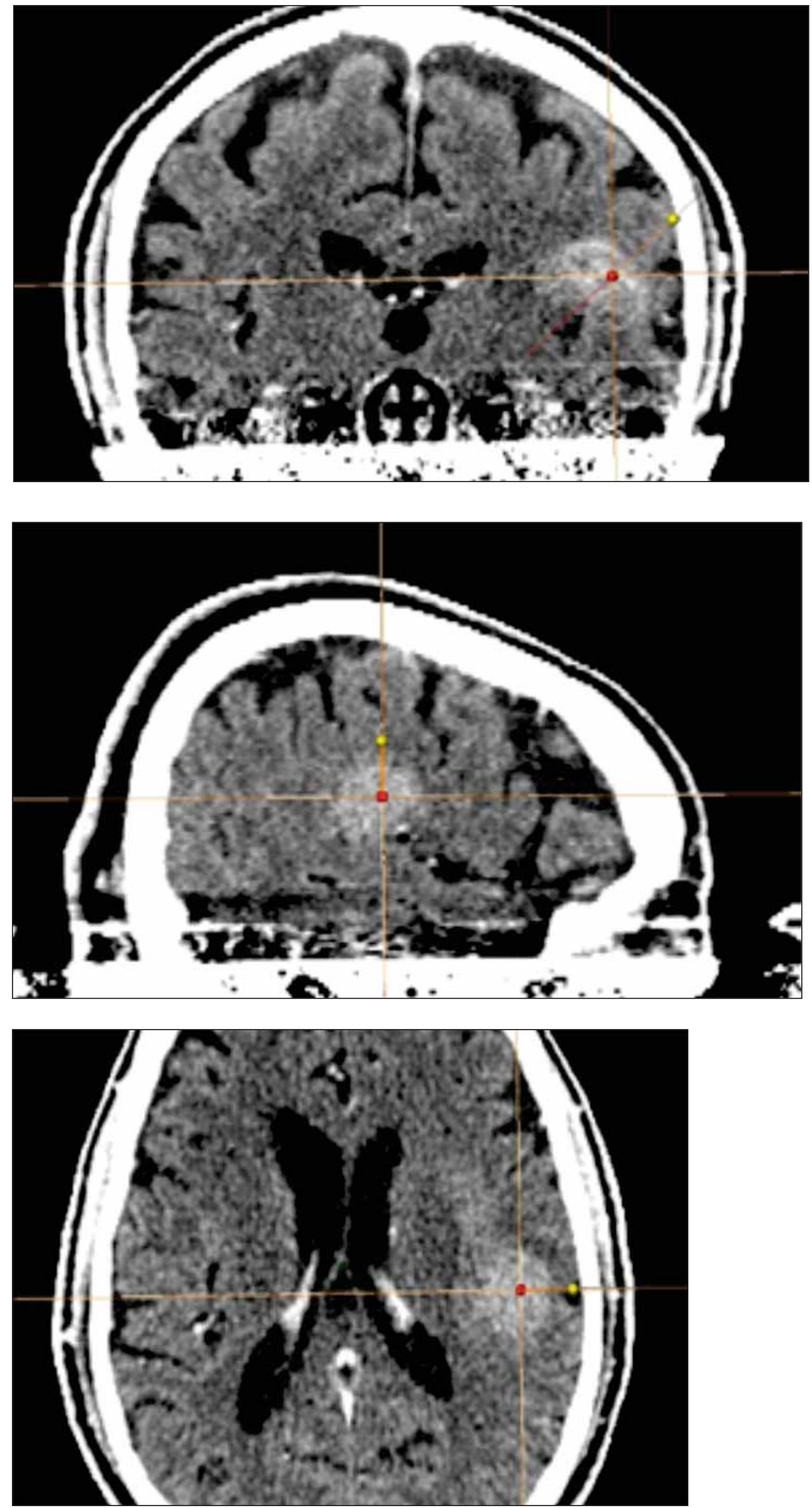

FIGURE 2. Stereotactic planning with CT images 
It is suggested that early started radiation therapy increases the period free of progression and seizures, despite the fact that it has no influence on overall survival, so it is of utmost importance to be initiated as soon as possible. Chemotherapy is used for holding back tumour progression (9).

For the classification of histopathological examination results we have used the 2016 WHO classification of primary brain tumours, in which protoplasmic and fibrillary astrocytomas are not subtypes of diffuse astrocytoma as they were until now, but are simply known as diffuse astrocytomas $(1,10)$. Because grade II gliomas have resembling invasive and malignant behaviour (although the gemistocytic type has a worse prognosis than the others) and pylocitic astrocytoma or grade I astrocytoma has an odd behaviour, we divided our results into grade I or grade II gliomas.

Follow-up is highly valuable for monitoring tumour progression or recurrence, clinical and neurological state and efficiency of the designated management. Follow-up was achieved at one year for all patients, 18 patients achieved a 2 year follow-up, 12 were observed at as much as 4 years and 10 were followed for 5 years.

\section{RESULTS}

Comparing to reported statistics, where individuals affected by low-grade gliomas have a median age of 35 years old and a sex ratio of 1,5 male to 1 female, our group of patients stands close, with a median age of 41 years old and a sex ratio of 1,3 to 1 . We also found that grade II gliomas are significantly more frequent (43) than grade I tumours (only 6), as found in other sources (11-13). Although most of the times seizures are cited to be the main reason for presentation $(14,15)$, most of our patients presented motor deficits as the cardinal symptom, seizures coming on second place, thus keeping their importance as one of the most frequent signs and symptoms that determined patients to search for medical care.

Of the 29 patients that have had performed a stereotactic guided biopsy, 26 had no deterioration after the procedure, while 3 experienced temporary mild deterioration with longer recovery to the initial state. 10 of these 29 patients presented seizures and complete seizure control (no reported episodes) was successful only for 6 patients on a one year follow-up. Fifteen of the patients had performed radiotherapy, and 14 were followed-up.

Of the 20 operated patients, 9 had a great post-operatory recovery with the remission of symptoms attributed to tumoral mass effect, the others remaining with the same neurological state as before surgery. Of the 8 patients who had seizures prior to surgery, complete control on one year follow-up was achieved in half of them. Fourteen patients of these twenty were diagnosed with grade II gliomas. Surgical resection and subsequent therapy (external beam, fractioned 54 Gy during 6 weeks) were performed.

Follow-up is highly valuable for monitoring tumour progression or recurrence, clinical and neurological state and efficiency of the designated management. At a one year follow-up, only 2 patients had a profound deterioration of their clinical state with a progression of the tumour. After initially being only biopsied, now surgical resection was performed, malignant transformation being suggested by MRS and confirmed by histopathological examination with the result of glioblastoma multiforme. Of the patients followed for 2 years, 6 had a deteriorated state, they suffered surgical resection and malignant transformation suggested by MRS was confirmed (4 glioblastoma multiforme and 2 ana-

TABLE 3. Metabolites levels in a grade // diffuse astrocytoma later confirmed by histopathological examination

\begin{tabular}{|l|r|r|r|r|r|r|r|} 
Position 1.1.1 Voxel Size: $11.0 \times 11.0 \times 11.0 \mathbf{m m}$ & \multicolumn{2}{c|}{ Real part } \\
\hline Metabolite & Peak Pos. & Peak Height & Half Width & Area & S.D. & Area(fit) & Height(fit) \\
\hline lip & 0.89 & 0.4 & 0.07233 & 35.1 & 0.19 & 35.06 & N/A \\
\hline lact & 1.26 & 0.1 & 0.06054 & 7.0 & 0.12 & 6.95 & N/A \\
\hline NAA & 1.96 & 1.1 & 0.08400 & 23.3 & 0.34 & 13.41 & 1.10 \\
\hline Cr & 2.95 & 1.0 & 0.09208 & 16.1 & 0.37 & 32.30 & 1.48 \\
\hline Cho & 3.16 & 1.4 & N/A & 20.1 & 0.58 & 44.09 & 1.93 \\
\hline ml & 3.48 & 0.3 & 0.03121 & 7.9 & 0.19 & 1.57 & 1.44 \\
\hline H2O & 4.27 & 0.1 & 0.04528 & 35.6 & 0.07 & 1.51 & 0.35 \\
\hline NAA/Cr & N/A & N/A & N/A & 1.4 & N/A & 0.42 & N/A \\
\hline Cho/Cr & N/A & N/A & N/A & 1.2 & N/A & 1.37 & N/A \\
\hline
\end{tabular}




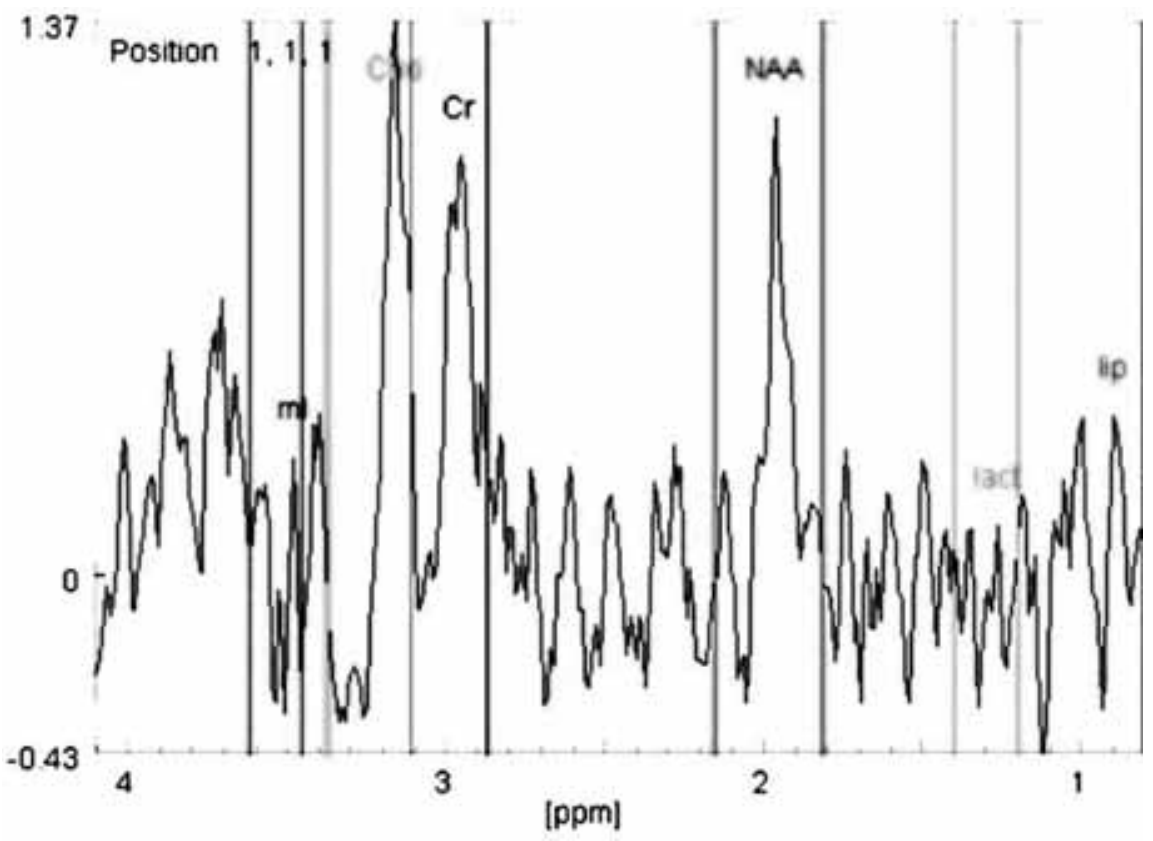

FIGURE 3. Graphic representation of metabolites levels in the same case as Table 3

plastic astrocytoma). For those who had a follow-up at 4 years, 4 had severe progression and surgical resection confirmed glioblastoma multiforme. After 5 years, 5 patients had severe progression of tumor, glioblastoma multiforme being confirmed after surgical resection. Eight patients who were biopsied and treated with radiotherapy had no changes on MRS.

All 22 patients who have been examined using Magnetic Resonance Spectroscopy were suspected for glioma according to their biochemical analysis. They were confirmed by histopathological examination of specimens obtained from stereotactic biopsy or surgery, 21 of which being grade II gliomas and just one grade I glioma. Biochemical findings on MRS consistent with low-grade glioma are represented by a relative decrease of NAA and rise in Choline levels (16-18) (Table 3).

\section{DISCUSSIONS}

There is no appropriate management for lowgrade gliomas. These type of tumours have a long natural history, with a slow growth rate and progression. Given these facts, if there are few to no benefits expected from surgical removal of the tumour, it is preferable that the physician adopt a more conservative management strategy with frequent follow-ups, and, at the most, confirm the diagnosis with a histopathological study of a needle-biopsy obtained specimen, prior to starting oncologic treatment (9). But this is where Magnetic Resonance Spectroscopy could come in useful. First of all, it is clear that it provides data for establishing the coordinates where the performed biopsy would deliver a sample which, analyzed from a histopathological point of view, gives an accurate diagnosis.

MRS is superior to other type of imaging studies because it highlights the metabolic differences of CNS lesions which can constitute key features in their accurate identification (19). Other imaging studies (CT, MRI etc.) only describe CNS lesions by their size, location, appearance (hyper/hypo density or intensity), post-contrast enhancement/ non-enhancement. Fig. 4 (images A, B, C) exemplifies a focal lesion with irregular margins, with mass effect and compressing neighboring structures, T1 hypo-intensity and T2 hyper-intensity, but without providing any clues for the identification of the histological type of the tumoral mass. In contrast, the MRS study shown in Table 3 and Fig. 3 suggests a diffuse astrocytoma, grade II WHO, confirmed by biopsy.

Today, we still need to have a histopathological exam, especially after the new classification of brain tumours that has appeared in June 2016 (WHO) which takes into account the molecular parameters of the tumour.

As the time passes, technology advances and we learn and discover more means of studying the inside of our bodies, obtaining specific data consistent with certain diagnoses. Also, training of the technicians executing imaging studies will probably make this relatively new area of interest more reliable and maybe everything we need for building 

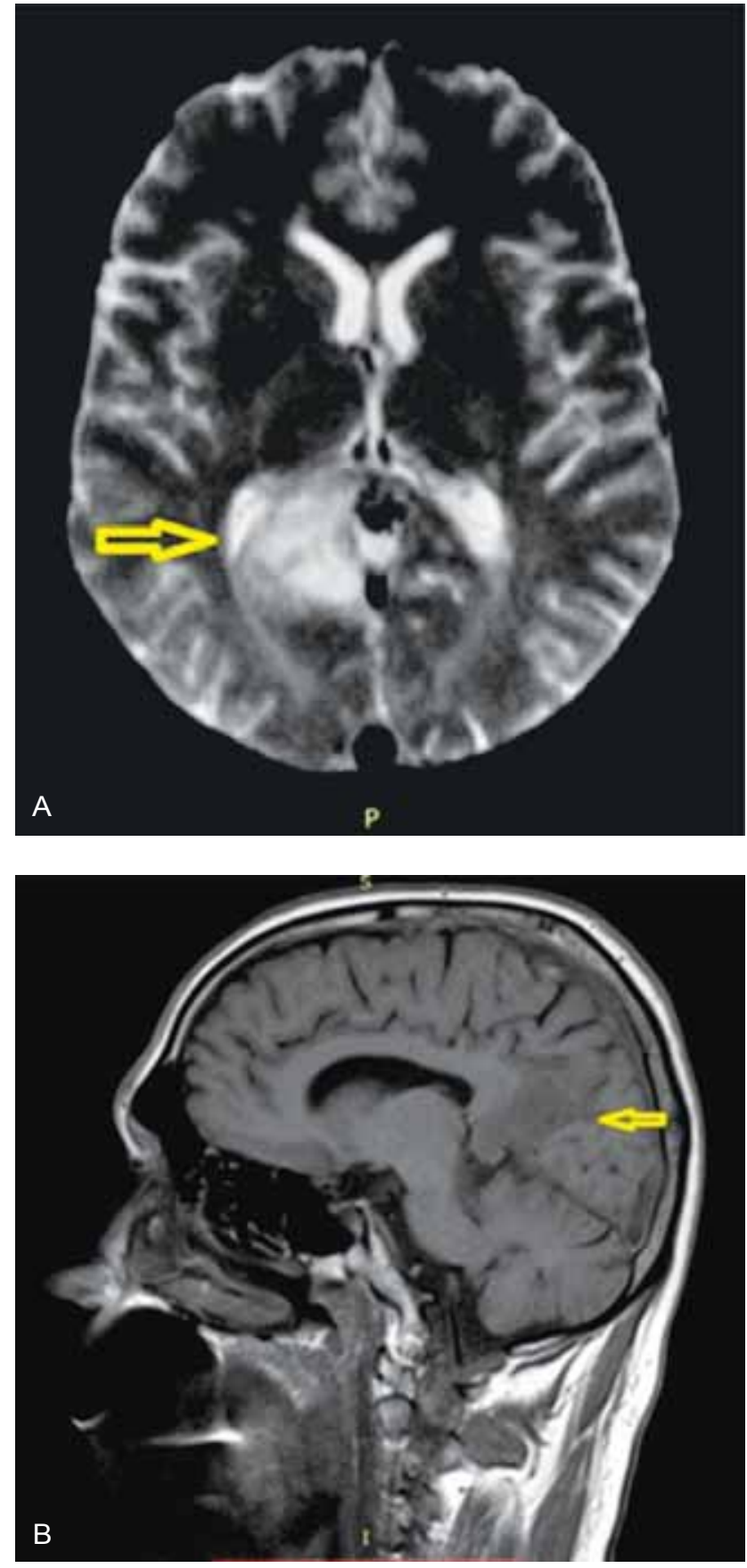

the basis of an optimal management for LGGs, where the comfort and well-being of the patient represent the creed we believe in.

The goals of low-grade glioma surgery are reducing tumoral mass (which is directly related with malignant transformation) and at the same time obtaining a specimen for diagnosis, improving neurological function, where this can be achieved and improving the control of seizures, if the case. For the best results, surgery should be performed in a setting with facilities including neurophysiology monitoring and mapping, intraoperative studies being also of great help (2). Surgical care should be

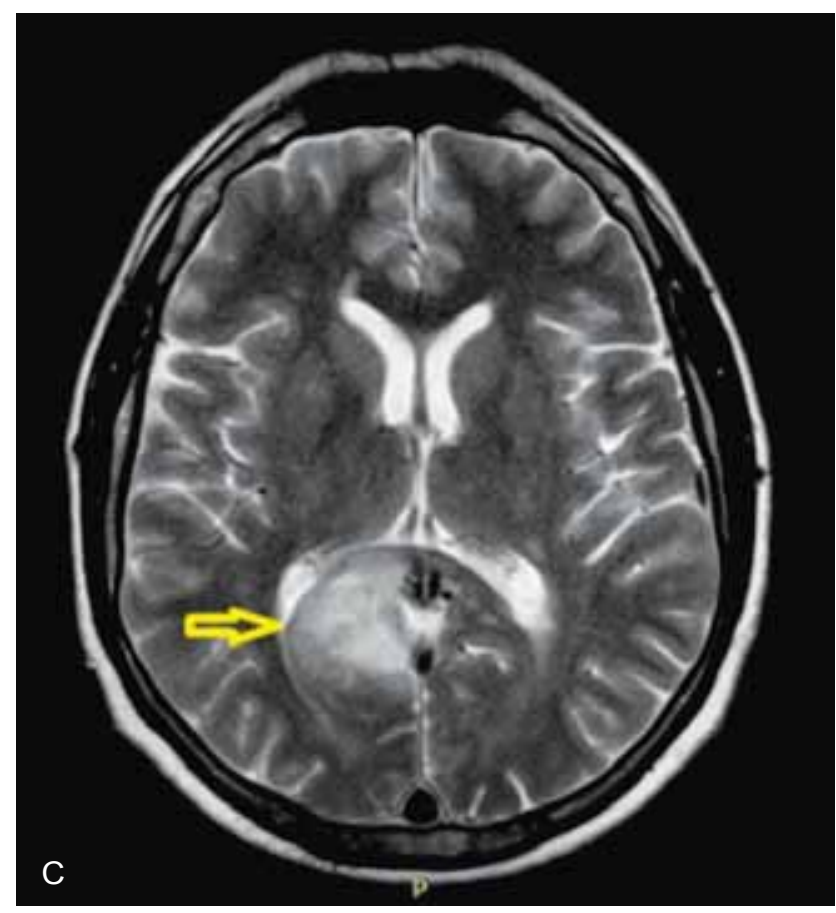

FIGURE 4. MRI showing a focal lesion in the right occipital medial grey matter, with a superior right parietal cortical projection and an inferior posterior right hippocampal projection with mass effect on the splenium of the corpus callosum on the right side, which is moved upwards and anterior, and compression of the posterior horn of the right lateral ventricle. The tumoral mass is indicated by the yellow arrows (axial diffusion - A, sagittal T1 - B, axial T2 - C)

taken into consideration as the main treatment for pilocityc astrocytomas, when there is a great danger of herniation or obstruction of CSF flow, but is doubtful for disseminated or multifocal tumours, or when located in eloquent brain (9).

When should surgery be performed? It should be delayed when minimal symptoms and small mass is present (clinical and imagistic surveillance including MRS). It should be performed immediately when there are extensive symptoms and a large mass. Subsequent resection shoult be made when there is a progressive mass or symptoms and MRS suggesting progression to a HGG. How much to resect? Maximal 
safe resection when feasible, although gross total resection is often not possible due to the infiltrative nature. Biopsy should be performed when resection is not feasible, minimal symptoms are present or a LGG is presumed (highly suggested by MRS).

Radiotherapy is delayed when there is a minimal mass or symptoms (including after resection) and in the case the MRS follow up suggests possible malignant transformation. Immediate radiotherapy is performed when a significant mass or symptoms are present, especially if only biopsy is performed or when "high risk" features are present (significant disease-related neurological symptoms, tumours crossing the midline, age $>40$ years, size $>5 \mathrm{~cm}$ ). Subsequent RT is rarely performed, suitable when recurrence/progression in new location. Typically the dose is 54 Gy, external beam, fractioned in 6 weeks. Post-operative management is vital for a good outcome. Stabilization of neurologic function in most patients has to do with receiving oncologic care, but also with the phenomenon of brain plasticity. But these also have their limits, which will probably be overcome in the future as new substances, procedures and techniques are developed $(2,12,14)$.
Limitations of MRS are the heterogeneity of tumor mass, which can affect the local concentration of metabolites, the device technical specifications and protocols used or voxel contamination from surrounding structures. These can hinder the establishment of absolute values that can be used as norms when executing MRS studies (5).

\section{CONCLUSIONS}

In our study, MR-Spectroscopy was a reliable tool for the accurate diagnosis of the histological grade of brain tumors. It was also of notable importance in the follow-up of the patients, as it is has some advantages: convenient for the patient, readily available, relatively easy to perform, rather reliable. There are a few impediments still to be overcome, which include physician training, MRI device technical specifications, standardization of data.

In the future, specific cases in thoroughly selected patients might have a greater benefit from this type of investigation, but considering molecular biology is the core for modern research in the domain of low-grade glioma, MRS will play a more complementary role.

Conflict of interest: none declared Financial support: none declared

\section{REFERENCES}

1. Louis D.N., Perry A., Reifenberger G. et al. The 2016 World Health Organization Classification of Tumors of the Central Nervous System: a summary. Acta Neuropathol. 2016; 131 (6): 803-20

2. Bello L., Dimeco F., Casaceli G., Gaini S.M. Surgical Management of Low-Grade Gliomas. In: Alfredo QuiñonesHinojosa. Schmideck and Sweet operative neurosurgical techniques, 6th Edition Vol 1. Philadelphia: Elsevier, 2012: 111-126

3. Al-Okaili R.N., Krejza J., Wanq S. et al. Advanced MR imaging techniques in the diagnosis of intraaxial brain tumors in adults. Radiographics. 2006; 26 Suppl 1: 173-89

4. Bradley W.G., Bydder G.M. Advanced MR Imaging Techniques. London: Martin Dunitz, 1997

5. Bulik M., Jancalek R., Vanicek J. et al. Potential of MR spectroscopy for assessment of glioma. Clinical Neurology and Neurosurgery. 2013; 115: 146-153

6. Choi C., Ganji S.K., DeBerardinis R.J. et al. 2-hydroxyglutarate detection by magnetic resonance spectroscopy in IDH-mutated patients with gliomas. Nat Med. 2012; 18(4): 624-9

7. Kim J.H., Chang K.H., Na D.G. et al. 3T H-MR Spectroscopy in Grading of Cerebral Gliomas: Comparison of Short and Intermediate Echo Time Sequences. AJNR. 2006; 27: $1412-1418$

8. Vuori K., Kankaanranta L., Häkkinen A.M. et al. Low-grade gliomas and focal cortical developmental malformations: differentiation with proton MR spectroscopy. Radiology. 2004; 230(3): 703-8

9. Greenberg M.S. Primary brain tumours In: Greenber MS Handbook of Neurosurgery, 7th Edition. Tampa Florida: Thieme, 2010: 590-697
10. Kros J.M. Grading of gliomas: the road from eminence to evidence. J Neuropathol Exp Neurol. 2011; 70(2): 101-9

11. Cavaliere R., Lopes M.B., Schiff D. Low-grade gliomas: an update on pathology and therapy. Lancet Neurol. 2005; 4(11): 760-70

12. Grier J.T., Batchelor T. Neuro-Oncology: Low-Grade Gliomas in Adults. The Oncologist. 2006; 11: 681-693

13. Macdonald D.R. Low-grade gliomas, mixed gliomas, and oligodendrogliomas. Semin Oncol. 1994; 21(2): 236-48

14. Brem H., Chiocca E.A., Sawaya R. Brain tumours. In: Brem $H$, Sawaya R, Chiocca EA, Burchiel KJ, Park TS, Ghatan S, Scott RM, Winn HR. Youmans Neurological Surgery Sixth Edition. Philadelphia PA: Elsevier, 2011: 1075-1086

15. Pouratian N., Schiff D. Management of Low-Grade Glioma. Current Neurology and Neuroscience Reports. 2010; 10(3): 224-231

16. Liu Z.L., Zhou Q., Zeng Q.S. et al. Noninvasive evaluation of cerebral glioma grade by using diffusion-weighted imagingguided single-voxel proton magnetic resonance spectroscopy. J Int Med Res. 2012; 40(1): 76-84

17. Sherif M.F., Salem F.M., Almahallawy M.A. et al. Role of magnetic resonance spectroscopy in differentiation between recurrence of glioma and post radiation injury. The Egyptian Journal of Radiology and Nuclear Medicine. 2014; 45(4): 1233-1240

18. Salzedo E., Cortes M., Melançon D. et al. Myoinositol trends in different types of brain lesions. Neuroradiol J. 2009; 22(1):16-21

19. Bertholdo D., Watcharakorn A., Castillo M. Brain proton MR spectroscopy: introduction and overview. Neuroimaging Clinics of NA. 2013; 23: 359-380 